\title{
AnÁlisis dimensional del Modelo NEOCLÁSICO DE CRECIMIENTO ECONÓMICO
}

\section{Miguel Alvarez Texocotitla ${ }^{1}$}

\section{Miguel David Alvarez, Hernández ${ }^{2}$}

\section{Shaní Eneida Alvarez Hernández ${ }^{3}$}

\section{Resumen}

El presente artículo de investigación tiene como propósito fundamental discutir los principios básicos del Análisis Dimensional en el contexto de la teoría económica, con el fin de aplicar dichos principios a las relaciones fundamentales que subyacen a la mayoría de los modelos de crecimiento económico. En particular, se utilizan las herramientas del Análisis Dimensional para evaluar la consistencia analítica del modelo Neoclásico. El análisis muestra que es necesaria una corrección a este modelo para que cumpla con el principio de homogeneidad dimensional.

Palabras clave: Análisis Dimensional, Economía Matemática, Modelos de Crecimiento Económico, Teoría Neoclásica.

\section{Abstract}

The fundamental purpose of the present paper is to introduce the basic principles of Dimensional Analysis in the context of the economic theory, in order to apply such principles to the fundamental relations that underlay most models of economic growth. In particular, instruments from Dimensional Analysis are used to evaluate the analytical consistency of the Neoclassical model. The analysis shows that an ad-

1 Doctor en Ciencias Sociales. Profesor-Investigador en la Universidad Autónoma Metropolitana, Unidad Iztapalapa.

2 MSc in Complex Systems Modelling por el King's College London.

3 Licenciada en Matemáticas por la Universidad Autónoma Metropolitana, Unidad Iztapalapa. 
justment to the model is required in such a way that the principle of dimensional homogeneity is satisfied.

Keywords: Dimensional Analysis, Mathematical Economics, Economic Growth Models, Neoclassical Theory.

\section{Introducción}

Para comprender el funcionamiento de un sistema económico se pueden establecer relaciones entre las variables fundamentales del sistema, las cuales son susceptibles de analizarse por medio de modelos matemáticos. Sin embargo, la modelación económica tiene exigencias analíticas que deben cumplirse, más si se pretende que el modelo tenga una correspondencia con la realidad y no sea éste una mera abstracción matemática. Las exigencias que analizaremos en el presente artículo se encuentran en el ámbito del análisis dimensional.

El Análisis Dimensional estudia las propiedades de las cantidades observables con dimensiones y de las relaciones matemáticas que las incorporan (Sonin, 2001). Este análisis se aplica en las ciencias naturales; de hecho, sus principios (dimensión, homogeneidad, medición y unidad) son claves en la formación del pensamiento científico ya que son parte de los principios básicos de la ciencia. El cumplimiento de los principios del Análisis Dimensional, y en especial el principio de la homogeneidad dimensional, es un requisito básico y necesario para realizar una apropiada modelación matemática, ya que permite comprobar ex post la consistencia dimensional de las relaciones matemáticas y señala las restricciones funcionales de las relaciones que se proponen entre las variables.

Sin embargo, en algunas disciplinas, como es el caso de la Economía, el concepto de dimensión y sus respectivos principios son prácticamente desconocidos. Son muy pocas las investigaciones que han hecho hincapié en las implicaciones del análisis dimensional en la disciplina económica. Entre ellas se tienen las de Grudzewski y Roslanowska (2013), cuyo libro de texto, proporciona un trabajo extenso sobre el uso y aplicaciones del análisis dimensional en la modelación 
económica; Barnett II (2004) analizó la consistencia dimensional de las funciones de producción, y en particular la función de Cobb-Douglas; Shone (2002) proporcionó una breve pero acertada introducción al uso de las dimensiones en los modelos económicos; Okishio (1982) presentó algunas aplicaciones del análisis dimensional en la teoría del valor-trabajo, y De Jong (1972), quien posiblemente fue el primero que señaló la importancia de la congruencia dimensional y la falta de ella en los modelos económicos. Tal insuficiencia de estudios es una de las razones que nos motiva a reconsiderar esta importante área del modelado matemático, ya que el Análisis Dimensional puede ofrecer nuevas perspectivas en cuanto a la forma en que se construyen los modelos económicos, y en algunos casos permitirá corregir los errores e insuficiencias que pudieran contener estos modelos.

Por otra parte, la investigación sobre las dimensiones representa una crítica fundamental a la metodología que se sigue en la modelación económica. Pese a que los modelos económicos puedan estar sustentados en una teoría matemática sólida, puede ser que en la construcción de los mismos no se contemplen las restricciones que existen al modelar fenómenos de distinta índole. Esta situación haría necesaria una revisión cuidadosa de los modelos económicos desde la perspectiva del Análisis Dimensional; en particular, de los modelos de crecimiento económico.

En este contexto, el presente estudio tiene como objetivo primordial presentar los principios básicos del Análisis Dimensional en el campo de la teoría económica, con el propósito de aplicar tales principios a las relaciones fundamentales que subyacen a los modelos de crecimiento económico. Específicamente, se utilizan las herramientas del Análisis Dimensional para evaluar la consistencia analítica del modelo Neoclá$\operatorname{sico}^{4}$. El análisis señala la necesidad de una corrección al modelo para que cumpla con el principio de homogeneidad dimensional.

4 La elección de este modelo en particular se puede justificar en tanto que constituye la estructura básica de numerosos modelos de crecimiento económico que han surgido posteriormente. 
Al final de la presente investigación, se ofrecen algunas reflexiones sobre la situación actual de la modelación económica, la pertinencia del análisis dimensional en la disciplina económica y las posibles líneas de investigación en torno a este tema.

\section{El Análisis Dimensional}

Las teorías científicas son construcciones lógico-abstractas que buscan explicar y predecir fenómenos, ya sean naturales, biológicos o sociales. La construcción de cualquier teoría científica inicia con la observación y la descripción de los fenómenos o eventos de interés para inferir (a partir de esas observaciones y descripciones) leyes, patrones y relaciones que representen al fenómeno de interés en la forma más simple y general posible (Einstein, 1933). Para cumplir dicho propósito la ciencia se vale del lenguaje matemático. Se podría aseverar que mientras una teoría no esté formulada con precisión en el lenguaje de la matemática, no es posible evaluar su relevancia ni su capacidad de predicción.

Sin embargo, el uso del lenguaje matemático tiene restricciones, ya que su utilización conlleva el cumplimiento de ciertas reglas. Estas reglas están relacionadas con las propiedades de las cantidades que son posibles de incluirse en un análisis cuantitativo ${ }^{5}$.

Por otro lado, el uso de las herramientas matemáticas es enriquecido con un axioma, el cual se deriva de una idea muy simple, pero fundamental: las relaciones matemáticas que se obtienen de una teoría científica deben relacionar fenómenos de una naturaleza similar tal que pueda establecer una relación de causa/efecto. Utilizando la jerga del Análisis Dimensional, podemos decir que las relaciones matemáticas de cualquier teoría deben ser equidimensionales.

La adopción de este axioma implica adoptar un criterio de validación. Una relación matemática es válida, en el contexto de la teoría, si

5 Aquí entendemos por cantidad a la descripción de alguna percepción sensorial o propiedad tangible. Por ejemplo, la noción de espacio (una propiedad física) es perceptible a través de múltiples cantidades, ya sea como distancias, áreas o volúmenes. 
en ambos lados de la igualdad (pensando en una ecuación algebraica, por ejemplo) se encuentran términos que sean iguales o similares. Sin embargo, decir sólo de forma intuitiva que dos observables son similares o iguales es insuficiente, pues, ¿Cómo definir qué es similar o qué es diferente? Es en este contexto que se introduce el concepto de dimensión, el cual permite manejar, con un formalismo matemático más fundamentado, el axioma de similaridad (White, 2011).

El axioma de similaridad, o el principio de Homogeneidad Dimensional, como se conoce en la literatura, expresa básicamente lo siguiente: si una relación matemática representa realmente una relación adecuada entre diversas observables de una cierta teoría, entonces dicha relación es homogénea, es decir, cada uno de los términos aditivos de dicha relación deberán ser equidimensionales.

\subsection{Aspectos básicos del Análisis Dimensional}

La presentación de los principios básicos del Análisis Dimensional exige definir algunos conceptos fundamentales. Se inicia por explicar qué se entiende por observable 6 .

Definición 1. Las cantidades que son posibles de cuantificar y de incluirse en una teoría consistente, i.e. son descriptibles por medio del lenguaje matemático, reciben la denominación de observables ${ }^{7}$.

Cada observable representa diferentes propiedades; por lo tanto, para poder determinar las posibles interrelaciones entre dichas observables es necesario establecer algún tipo de estructura. Básicamente, si se quiere comparar dos observables distintas, ambas deben tener definidas una operación de causalidad o equivalencia.

6 Esta sección no pretende dar una explicación completa y exhaustiva del Análisis Dimensional, para una descripción más detallada se recomienda revisar las referencias bibliográficas.

7 Balaguer (2013), Bunge (1971), Carlson (1979), Sonin (2001) y White (2011) utilizan la denominación observable física. Sin embargo, en este trabajo abandonamos el adjetivo físico para recalcar que las reglas del Análisis Dimensional se aplican de igual forma a las observables económicas. 
Definición 2. Denotemos por $O$ al conjunto de todas las posibles observables que puedan existir dentro de una teoría:

$$
0 \equiv\{A, B, C, \ldots\}
$$

Dicho conjunto posee la siguiente estructura:

i) Una operación de causalidad $(=, \neq)$, que permita determinar una relación de orden (la igualdad o la desigualdad) entre dos observables, e.g. $A=B o A \neq B$.

Se debe enfatizar que la operación de causalidad tiene su justificación en la experiencia real, ya que la acción de comparar dos observables está relacionada cercanamente con la posibilidad de realizar manipulaciones de objetos o eventos que permitan realizar alguna comparación o medición que determine la causa/efecto (Sonin, 2001). Por ejemplo, no existe manera alguna de comparar una observable cardinal (que represente la propiedad de cantidad) con una observable temporal (que represente la propiedad de tiempo), ya que en principio no es posible definir una relación de equivalencia que proporcione un resultado con sentido real y físico.

Aparte de la operación de causalidad, otras operaciones matemáticas pueden ser definidas para las observables. Sin embargo, para ello es necesario definir el concepto de dimensión (Bunge, 1971).

Definición 3. Dimensión es la descripción cualitativa de alguna propiedad física, la cual puede compartir diferentes tipos de observables. Sea $\mathrm{D}$ el conjunto de todas las posibles dimensiones, dentro de una teoría, asociadas a $O$ :

$$
\mathrm{D} \equiv\{\mathrm{A}, \mathrm{B}, \mathrm{C}, \ldots\}
$$

Este conjunto debe poseer la estructura de un grupo conmutativo, la cual está determinada por:

i) Una operación binaria (*), e.g. $\mathrm{A} * \mathrm{~B}=\mathrm{C}$

ii) Un elemento neutro $\left(1_{D}\right)$, e.g. $\mathrm{A} * 1_{D}=\mathrm{A}$

iii)Un elemento inverso $\left(\mathrm{A}^{-1}\right)$, e.g. $\mathrm{A} * \mathrm{~A}^{-1}=1_{D}$ 
$\mathrm{Y}$ debe satisfacer las condiciones siguientes:

iv) La operación es asociativa, e.g. $A *(B * C)=(A * B) * C$

v) La operación es conmutativa, e.g. $\mathrm{A} * \mathrm{~B}=\mathrm{B} * \mathrm{~A}$

Definición 4. La asignación de dimensiones a las observables se realiza por medio de un operador [ ]: $O \rightarrow D$, el cual se denomina función dimensión. Esta función asigna a cada elemento de $O$ un correspondiente elemento en $\mathrm{D}$, de tal manera que

i) Si $\boldsymbol{A}, \boldsymbol{B} \in \boldsymbol{O}, A, B \in D$, y $\alpha, \beta$ son números reales, entonces $\left[\alpha \boldsymbol{A}^{\alpha} \beta \boldsymbol{B}^{\beta}\right]=\alpha\left[\boldsymbol{A}^{\alpha}\right] \beta\left[\boldsymbol{B}^{\beta}\right]=\alpha A^{\alpha} \beta B^{\beta}$

ii) Si $\alpha \in \mathbb{R}$, entonces $[\alpha]=1_{\mathrm{D}}$

Se puede ejemplificar el uso de la definición 4 . Considérese una tasa de interés $\rho$, y la dimensión asociada a dicha observable es $\mathrm{T}^{-1}$; entonces el resultado de aplicar la función dimensión sería $[\rho]=\mathrm{T}^{-1}$. Si se tuviera otro objeto que no perteneciera al conjunto $O$, por ejemplo, cualquier valor numérico $r$, entonces la función dimensión devolvería el elemento neutro o adimensional, $[r]=1_{D}$. Al igual que las dimensiones, la función dimensión tiene asociada la misma álgebra.

Las anteriores definiciones permiten una definición más formal de observable.

Definición 5. Una observable $A \in O$ se define como el producto de un valor numérico $\alpha \in \mathbb{R}$, y de una dimensión $A \in D$ :

$$
A \equiv \alpha^{*}[A]
$$

Asimismo, con las definiciones anteriores se presenta ahora el concepto de equidimensionalidad y la condición de equivalencia entre observables.

Definición 6. Sean $A, B \in O$, y $C \in D$. Entonces, $A$ y $B$ se dice que son equidimensionales si y sólo si tienen la misma dimensión, es decir:

$$
[A]=C=[B]
$$


Definición 7. Sean $\boldsymbol{A}, \boldsymbol{B} \in \boldsymbol{O}$ dos observables. Sean además $\alpha, \beta$ los valores numéricos y $A, B$ las dimensiones asociadas a $\boldsymbol{A}, \boldsymbol{B}$ respectivamente. Entonces, $A$ y $B$ se dice que son equivalentes (iguales), si y sólo si tienen el mismo valor numérico y la misma dimensión. Es decir:

$$
\mathbf{A}=\boldsymbol{B} \text { si y sólo si } \alpha=\beta \text { y }[\boldsymbol{A}]=[\boldsymbol{B}]
$$

En consecuencia:

i) Las observables tienen definida una operación binaria $(* /)$ de producto/división.

ii) Sólo las observables equidimensionales tienen definida una operación binaria $( \pm)$ de adición/resta.

iii)Los argumentos de funciones trascendentales, e.g exponenciales o logaritmos, deben ser estrictamente términos adimensionales.

iv)El cociente de dos observables equidimensionales da como resultado una observable adimensional.

\subsection{El principio de Homogeneidad Dimensional}

Las teorías científicas normalmente involucran dos variedades de ecuaciones: matemáticas y observables (Sonin, 2001). Las ecuaciones matemáticas comprenden solamente valores numéricos u otras entidades matemáticas que no poseen ningún significado real innato, lo cual no implica que aun así no se puedan explorar y estudiar las relaciones y propiedades que puedan surgir de dichas ecuaciones.

Por su parte, las ecuaciones observables surgen primordialmente de la observación y los datos experimentales. La importancia de estas ecuaciones estriba en que relacionan de forma balanceada un conjunto de observables contra otro conjunto de observables a través del signo de igualdad matemática (Baiocchi, 2012).

Debido a que las ecuaciones observables contienen cantidades observables, las cuales necesariamente cargan un significado real, es necesario cuidar que la ecuación que se está proponiendo esté debidamente balan- 
ceada. En consecuencia, el uso de las dimensiones proporciona esencialmente el criterio de balance en las ecuaciones. La siguiente definición permite definir la igualdad (balance) dimensional de una ecuación.

Definición 8. Una ecuación es equidimensional si y sólo si cada uno de las observables aditivas que la componen es equidimensional.

En otras palabras, una ecuación observable es válida únicamente si es una ecuación equidimensional, esto es, si todos los términos involucrados en la ecuación son equidimensionales. Esta idea sencilla marca el principio más fundamental del análisis dimensional y es tal su importancia que la idea se eleva a nivel de axioma (Sonin, 2001). Axioma que se presenta a continuación.

Axioma o principio de la Homogeneidad Dimensional. Una ecuación que involucre observables es válida y tiene un significado real en el contexto de la teoría, si y sólo si cada una de las observables involucradas en la ecuación son equidimensionales entre sí.

El origen de este axioma antecede a la formalización del análisis dimensional, ya que para calcular un resultado proveniente de alguna ecuación se debe tener una coincidencia entre el resultado y los términos u observables que originaron dicho resultado, es decir, en principio sólo se puede establecer una relación causa/efecto entre fenómenos de la misma índole (Macagno, 1971). Como resultado del axioma se deriva el siguiente teorema.

Teorema. La validez numérica de una ecuación que involucre observables existe si y sólo si los términos a ambos lados de la igualdad son equidimensionales.

Considérese el siguiente ejemplo. Una teoría considera que las únicas observables relevantes son: el número de máquinas disponibles $X$, el número total de trabajadores $P$, la cantidad de trabajadores inactivos $P_{I}$ y el número de máquinas producidas $Y$. Se propone que el número de máquinas producidas está dado por la siguiente ecuación:

$$
Y=X+\left(P-P_{I}\right)
$$


Por el principio de homogeneidad dimensional, sólo podemos calcular un resultado válido para $Y$ si y solo si $X, P, P_{I}, Y$ son equidimensionales. El término $X$ es equidimensional con $Y$, ya que ambos representan dos cantidades de una misma observable, por lo tanto, sí es posible establecer una relación de igualdad entre ambos términos. Los términos $P$ y $P_{I}$ son equidimensionales entre sí y, por ende, la sustracción de ambos sí tiene sentido físico. Sin embargo, $P$ y $P_{I}$ no son equidimensionales respecto a $X$ o $Y$, entonces no tiene sentido establecer una relación de suma entre dichas observables, tal y como se expresa en la ecuación. Visto desde el punto de vista dimensional, la ecuación $Y$ carece de todo sentido y significado real con las actuales observables involucradas, ya que no hay manera alguna de establecer el significado de restar o sumar cantidad de máquinas con número de trabajadores. La única manera de volver válida la ecuación $Y$ es si se incorporan diferentes observables o términos adicionales que homogeneice las dimensiones de la ecuación.

\section{El Análisis Dimensional en Economía}

En esta sección se aplican los principios del Análisis Dimensional a la teoría económica, y se analizan algunas relaciones económicas fundamentales que subyacen a los modelos de crecimiento económico. Se muestra que dichas relaciones adolecen de inconsistencias desde el punto de vista del análisis dimensional, las cuales son posibles de corregir, pero a costa de enfrentar nuevas implicaciones analíticas.

El Análisis Dimensional tiene sus raíces en la naturaleza de los artificios que se han construido con el fin de describir el mundo físico y explicar su funcionamiento en términos cuantitativos; su origen puede ser rastreado desde las formulaciones geométricas que realizaban los griegos matemáticos de la antigüedad. Sin embargo, su fundamento moderno comenzó a ser establecido hace dos siglos, y fue principalmente en el siglo XX que se adoptó su uso de forma extensa en las ciencias naturales.

La primera persona que escribió acerca del problema de las unidades y las dimensiones (enfocado esencialmente a los modelos y teorías de 
la Física) fue Leonard Euler en 1765. Por su parte, Joseph Fourier en su libro The Analytic Theory of Heat, publicado en 1822, remarcaba lo que ahora se conoce como el principio de la Homogeneidad Dimensional, e incluso desarrolló algunas de las reglas que se utilizan actualmente en el análisis dimensional. Posteriormente, no ocurrieron avances significativos en el área hasta la publicación del libro de Lord Rayleigh en 1877, The Theory of Sound, el cual proponía un método muy similar al método moderno de dimensiones y daba numerosos ejemplos de cómo utilizar el Análisis Dimensional. No obstante, de contribuciones anteriores la más notoria, que estableció el método y las reglas actuales del Análisis Dimensional, es la de E. Buckingham, quien en 1914 implementó la formalización más utilizada del Análisis Dimensional (Macagno, 1971).

Por lo que respecta al campo de la Economía, el concepto de dimensión y sus respectivos principios son prácticamente desconocidos. Pocos investigadores han analizado las implicaciones del Análisis Dimensional en Economía; entre éstos se encuentran Grudzewski y Roslanowska (2013); Barnett II (2004); Shone (2002); Okishio (1982), y De Jong (1972).

\subsection{Stocks, flujos y dimensiones económicas}

Shone (2002) puntualiza la distinción entre dos tipos principales de observables económicas, las observables de tipo stock (acervo) y las observables de tipo flujo. Las observables de tipo stock hacen referencia al valor de una cierta cantidad económica en una fecha determinada -es decir en un punto exacto del tiempo-. Supongamos que nuestra observable económica es la cantidad de dinero $(M s)$ en la Economía. Esta observable tendría un valor definido para cada instante de tiempo, por ejemplo al 31 de diciembre, y tendrá otro valor definido $\left(M^{\prime} s\right)$ para cualquier otro instante de tiempo anterior o posterior. Es decir, el carácter dimensional de un observable stock es independiente de cualquier intervalo de tiempo. 
Por el contrario, una observable de tipo flujo hace referencia al valor total o promedio de una cierta cantidad económica en un intervalo de tiempo. Si consideramos la demanda de bienes $(D)$ en un periodo de tiempo determinado, en un año, por ejemplo, dicha observable es de tipo flujo, ya que estamos hablando de una observable que se distribuye en el tiempo, y por ende su valor está definido en dicho intervalo. Es decir, las observables de tipo flujo tienen una dependencia dimensional directa con el tiempo.

Si suponemos que las observables de stock y flujo están representadas por funciones continuas y diferenciables, la relación que existe entre un stock y un flujo se puede representar matemáticamente como ${ }^{8}$ :

$$
\frac{d Q(t)}{d t}=F(t)
$$

Donde $Q(t)$ es la función asociada a una observable tipo stock y $F(t)$ es la función asociada a la observable tipo flujo. Por lo tanto, dado que la derivada es en sí un operador matemático que depende dimensionalmente del tiempo, cualquier observable tipo stock podrá transformarse por medio de dicho operador a una observable de tipo flujo ${ }^{9}$.

Otra forma de ver la diferencia entre un stock y un flujo es pensar a $F(\mathrm{t})$ como el equivalente a una tasa de cambio (es decir una velocidad) de una determinada cantidad o stock, en este caso $Q(t)$. En consecuencia, dada su diferencia, la única manera en que ambas observables pudieran ser iguales, i.e. equidimensionales, será a través de la utilización de otro término que homogeneiza las dimensiones de la relación. En este caso, el término que homogeneiza la relación es el operador derivada, ya que dicho operador transforma $Q(t)$ de un stock a un flujo $F(t)$.

8 Esta definición también puede expresarse en su equivalente en tiempo discreto.

9 Nótese que a pesar de que las variables sean funciones del tiempo no implica que incorporen alguna dimensión temporal. Sólo cuando está incorporada explícitamente alguna observable temporal, como es el caso del operador derivada, las dimensiones de la variable incorporan una dimensión temporal (T). 
Por lo tanto, un stock y un flujo no son equidimensionales, es decir, no pueden ser comparados, igualados, sumados o restados, ya que dichas operaciones no están definidas para operar entre observables no equidimensionales. Esta diferencia en las dimensiones de los stocks y de los flujos impone restricciones que se deben tomar en consideración, sobre todo cuando se combinan observables de diferente naturaleza.

Una vez que se han discutido los conceptos de stock y flujo, corresponde considerar las dimensiones en Economía.

¿Cómo catalogar las diferentes dimensiones de las observables económicas? Fuera del campo de la Física, la elección de las dimensiones fundamentales no es tan clara, y depende mucho del área de aplicación y de la teoría utilizada. Por ejemplo, en el caso de la teoría económica, parece ser suficiente tener como dimensiones fundamentales el tiempo $(T)$, el valor monetario $(M)$, la cantidad de elementos $(Q)$ y la utilidad $(U)^{10}$. Este conjunto de dimensiones presenta un buen punto de partida para incorporar la cuestión de las dimensiones en los modelos de crecimiento, ya que no parece haber características económicas más fundamentales que las representadas por estas cuatro dimensiones ${ }^{11}$. Por esta razón se utilizarán a lo largo del presente artículo. En el Cuadro 1 se pueden observar y comparar las dimensiones utilizadas en Economía y en Física.

10 Dicho conjunto de dimensiones se propone en Shone (2002).

11 Sin embargo, la justificación de por qué dicho conjunto es adecuado y suficiente es una cuestión que aún está por determinar. 


\section{Cuadro 1. Propuesta de dimensiones fundamentales en Economía}

\begin{tabular}{|l|c|l|}
\hline \multicolumn{3}{|c|}{ Dimensiones económicas fundamentales } \\
\hline \multicolumn{1}{|c|}{ Observable } & Dimensión & Unidades de medición \\
\hline Valor Monetario & $M$ & dólares, onza de oro, ... \\
\hline $\begin{array}{l}\text { Cantidad de } \\
\text { elementos }\end{array}$ & $Q_{X}$ & $\begin{array}{l}\text { número bienes, núme- } \\
\text { ro de agentes, ... }\end{array}$ \\
\hline Tiempo & $T$ & segundo, años, ... \\
\hline Utilidad $^{12}$ & $U$ & unidades de utilidad? \\
\hline \multicolumn{2}{|c|}{ Dimensiones físicas fundamentales } \\
\hline \multicolumn{1}{|c|}{ Observable } & Dimensión & Unidades de medición \\
\hline Masa & $M$ & gramo, libras, ... \\
\hline Longitud & $L$ & metro, pulgada, ... \\
\hline Tiempo & $T$ & segundo, años, ... \\
\hline Entropía & Bit & shanon, nat, ... \\
\hline
\end{tabular}

Fuente: Elaboración propia.

\subsection{El Análisis Dimensional en los modelos de crecimiento económico}

En esta sección se analizan dos identidades que son fundamentales en los modelos de crecimiento económico: la identidad de ingreso del hogar representativo y la identidad de beneficio de la empresa representativa. De ambas identidades se derivan las relaciones que determinan la dinámica de la producción y de la acumulación de capital físico.

Considérese el caso de un modelo de crecimiento económico de una economía cerrada que contiene sólo dos agentes económicos, un hogar y una empresa ${ }^{13}$. Ambos intercambian en el mercado un solo bien de consumo/producción, el cual se denota como capital físico. Este único

12 Se advierte que las unidades correspondientes a la utilidad llevan una interrogación para remarcar el hecho que no existe en sí alguna unidad de medición asociada a U.

13 Ambos se pueden considerar como los agregados o representantes de todos los demás agentes del sistema, por lo tanto, también reciben los nombres de hogar y empresa representativos. 
bien representativo se utiliza simultáneamente como un insumo de producción y como un bien de consumo ${ }^{14}$.

Además de la existencia de un solo bien, se asume que existe otro factor económico, el cual afecta únicamente al proceso productivo (es decir, sólo funciona como un insumo de producción). A este factor se le denomina trabajo. La combinación de ambos factores (capital físico y trabajo) tiene lugar en el proceso productivo y desemboca en la producción de más capital físico, el cual puede o no utilizarse nuevamente en el proceso productivo.

Siguiendo las hipótesis convencionales de los modelos de crecimiento económico, se considera que la estructura del mercado donde participan ambos agentes es de competencia perfecta. Es decir, el hogar y la empresa actúan como agentes que toman sus decisiones independientemente de los precios de mercado asociados a los insumos de producción y del bien representativo.

Por otra parte, se asume que el hogar es el propietario de los factores de producción y su ingreso $Y_{H}$, por la renta de dichos insumos a la empresa, estará dado por:

$$
Y_{H}(\mathrm{t})=w_{k}(\mathrm{t}) K_{H}(\mathrm{t})+w_{L}(\mathrm{t}) L_{H}(\mathrm{t})
$$

Donde $w_{K} \mathrm{y} w_{L}$ denotan los precios de renta del capital físico $K_{H} \mathrm{y}$ del trabajo $L_{H}$ (Acemoglu, 2009:32).

Considerando las dimensiones de cada término aditivo se analiza ahora la congruencia dimensional de la Eq. (2). El capital físico representa la cantidad de bienes que existen en un instante de tiempo, es decir es una observable de tipo stock. El trabajo, a diferencia del capital físico, representa una observable de tipo flujo, ya que se mide en términos de unidades de trabajo utilizadas en un intervalo de tiem-

$14 \mathrm{Al}$ igual que en el caso del hogar y la empresa representativos, este bien se puede considerar como el agregado de todos los demás bienes del sistema. 
po (Pindyck y Rubinfeld, 2009:219). En consecuencia, las dimensiones asociadas a $K_{H}$ y $L_{H}$ son:

$$
\left[K_{H}\right]=Q_{K}, \quad\left[L_{H}\right]=\frac{Q_{L}}{T}
$$

Con $Q_{K}$ simbolizando la dimensión de cantidad de capital físico, $Q_{L}$ la dimensión de cantidad de trabajo y $T$ la dimensión de tiempo.

El ingreso $Y_{H}$ se expresa en términos de unidades monetarias recibidas en un intervalo de tiempo, e.g. el ingreso que reporta un individuo por la renta de un servicio puede ser expresado en términos de dólares/mensuales. Por lo tanto $\left[Y_{H}\right]=\frac{M}{T}$. Sin embargo, en el contexto del modelo que se desarrolla, no existe un Banco Central ni tampoco una unidad monetaria. Es decir, estamos considerando una economía no monetaria de intercambio de bienes por bienes. En consecuencia, el ingreso es una observable económica de tipo flujo de bienes de capital físico, i.e. estamos haciendo el reemplazo $M \rightarrow Q_{K}$, y por lo tanto sus dimensiones se expresan como:

$$
\left[Y_{H}\right]=\frac{M}{T}=\frac{Q_{K}}{T}
$$

Los precios $w_{K}$ y $w_{L}$ representan cuantas unidades monetarias equivalen a una unidad de capital físico o a una unidad de trabajo, pero en el contexto de una economía no monetaria, los precios sólo representan cuantas unidades de capital físico equivalen a una unidad de trabajo. Es decir ${ }^{15}$ :

$$
\left[w_{K}\right]=\frac{M}{Q_{K}}=\frac{Q_{K}}{Q_{K}}=1_{D}, \quad\left[w_{L}\right]=\frac{M}{Q_{L}}=\frac{Q_{K}}{Q_{L}}
$$

En consecuencia, reescribiendo la Eq. (2) en términos de las dimensiones de cada variable se obtiene:

15 Nótese que, bajo el supuesto de una Economía de intercambio de bienes por bienes, el precio del capital físico se vuelve una cantidad adimensional. 


$$
\begin{gathered}
{\left[Y_{H}\right]=\left[w_{K}\right]\left[K_{H}\right]+\left[w_{L}\right]\left[L_{H}\right]=\left(1_{D}\right)\left(Q_{K}\right)+\left(\frac{Q_{K}}{Q_{L}}\right)\left(\frac{Q_{L}}{T}\right)} \\
\left(\frac{Q_{K}}{T}\right) \neq\left(Q_{K}\right)+\left(\frac{Q_{K}}{T}\right)
\end{gathered}
$$

Por lo tanto, comparando las dimensiones de cada término aditivo se encuentra:

$$
\left[Y_{H}\right] \neq\left[w_{K}\right]\left[K_{H}\right], \quad\left[Y_{H}\right]=\left[w_{L}\right]\left[L_{H}\right]
$$

Y esto muestra el primer resultado importante del análisis dimensional de la identidad ingreso, Eq. (2): la relación que describe el ingreso del hogar no es dimensionalmente congruente, ya que no se cumple que en ambos lados de la igualdad se encuentren las mismas dimensiones (Ver Sección 1). ¿De dónde proviene este fallo?

La incongruencia dimensional se genera por la distinta naturaleza dimensional del capital físico y del trabajo. En la Eq. (2) se asume que ambos factores, capital físico y trabajo, pueden sumarse entre sí simplemente porque ambos factores se ven multiplicados por sus respectivos precios, sin embargo, ambos factores tienen una naturaleza distinta, ya que uno describe un stock mientras que el otro factor describe a un flujo.

Se examina ahora qué pasa en el caso de la empresa. Teóricamente se considera que la empresa actúa conforme a la maximización de su beneficio. Asimismo, se supone que el proceso productivo depende solamente de los insumos de producción (capital físico y trabajo) y que dicho proceso puede ser descrito mediante una función de producción $F\left(K_{F} ; L_{F}\right)$. Es decir:

$$
Y_{F}=F\left(K_{F} ; L_{F}\right)
$$

Por lo tanto, el beneficio de la empresa estará dado por:

$$
\pi=F\left(K_{F} ; L_{F}\right)-\left(w_{K}(t) K_{F}(t)+w_{L}(t) L_{F}(t)\right)
$$


Donde $\pi$ denota el beneficio de la empresa reportado en unidades de capital físico obtenidas en un intervalo de tiempo, $Y_{F}$ indica la producción realizada por la empresa expresada de igual forma en unidades de capital físico por periodo de tiempo, $w_{K}$ y $w_{L}$ son los precios del capital físico y del trabajo, $K_{F}$ representa la cantidad de capital físico utilizado por la empresa y $L_{F}$ la cantidad de unidades de trabajo utilizadas en el intervalo de tiempo de producción.

Al igual que antes, se reescribe la Eq. (5) en términos de las dimensiones de cada término:

$$
\begin{gathered}
{[\pi]=\left[Y_{F}\right]=\frac{Q_{K}}{T}, \quad\left[F\left(K_{F}, L_{F}\right)\right]=\frac{Q_{K}}{T}, \quad\left[K_{F}\right]=Q_{K}, \quad\left[L_{F}\right]=\frac{Q_{L}}{T}} \\
{\left[w_{K}\right]=1_{D}, \quad\left[w_{L}\right]=\frac{Q_{K}}{Q_{L}}}
\end{gathered}
$$

Desarrollando,

$$
\begin{gathered}
{[\pi]=\left[F\left(K_{F} ; L_{F}\right)-\left(w_{K} K_{F}+w_{L} L_{F}\right)\right]} \\
\left(\frac{Q_{K}}{T}\right)=\left(\left(\frac{Q_{K}}{T}\right)-\left(1_{D}\right)\left(Q_{K}\right)+\left(\frac{Q_{K}}{Q_{L}}\right)\left(\frac{Q_{L}}{T}\right)\right)=\left(\frac{Q_{K}}{T}\right)-\left(Q_{K}\right)+\left(\frac{Q_{K}}{T}\right)
\end{gathered}
$$

$\mathrm{Y}$, en consecuencia, se obtiene una inconsistencia en las dimensiones de la expresión Eq. (5):

$$
[\pi] \neq\left[w_{K} K_{F}\right]
$$

Al igual que la inconsistencia de la Eq. (2), ésta proviene del hecho de que se comparan (se suman en este caso) dos términos de distinta naturaleza; se compara una cantidad de tipo stock (capital físico) con una cantidad de tipo flujo (trabajo).

¿Cómo corregir esta inconsistencia dimensional? Hay dos alternativas de solución. La primera es considerar una interpretación distinta del capital físico para que asuma las dimensiones que corresponden a un flujo $\left([K]=Q_{K} / T\right)$, y de esta forma el término $w_{K} K$ adquiera las di- 
mensiones correctas. Sin embargo, es difícil justificar económicamente una interpretación distinta de $K$ cuando es un componente de la identidad ingreso.

La segunda opción es considerar una interpretación distinta del término $w_{K}$. Supongamos que en lugar de $w_{K}$ se introduce una tasa de rentabilidad (rendimiento), es decir ( $w_{K} \rightarrow r_{K}$ ), la cual representaría cuánto capital físico es dado por una unidad de capital físico en un intervalo de tiempo determinado.

Por otra parte, hay que tener en cuenta que el capital físico puede incorporarse o salir del mercado de los insumos productivos. Es decir, se incorpora al mercado nuevo capital, pero de igual forma se pierde capital cuando éste sale del mercado por desgaste, destrucción, avería, o por volverse obsoleto. Bajo esas consideraciones también se justifica el introducir una tasa de depreciación del capital, la cual se asumirá constante por simplicidad, i.e. $(\dot{K}(t) / K(t))=-\delta$ (Barro \& Sala-i-Martin, 2009 , pp. 32). Por lo tanto, si se introducen dichos cambios en la Eq. (2) y en la Eq. (5), considerando que las dimensiones de $r_{K}$ y $\delta$ son:

$$
\left[r_{K}\right]=\frac{Q_{K}}{Q_{K} T}=\frac{1}{T}=[\delta]
$$

Encontramos que la inconsistencia dimensional de la Eq. (2) y la Eq. (5) queda resuelta, como puede observarse a continuación ${ }^{16}$.

$$
\begin{gathered}
Y=r_{K}(t) K(t)+w_{L}(t) L(t) \\
{[Y]=\frac{Q_{K}}{T}=\left[r_{K} K\right]=\left[w_{L} L\right]} \\
\pi=F(K ; L)-\left(\left(r_{K}(t)+\delta\right) K(t)+w_{L}(t) L(t)\right) \\
{[\pi]=\frac{Q_{K}}{T}=[F(K ; L)]=\left[r_{K} K+w_{L} L\right]}
\end{gathered}
$$

16 Se han eliminado los subíndices de K y L, ya que se considera que el mercado se encuentra en situación de equilibrio. Es decir, las cantidades de capital y trabajo que requiere la empresa son iguales a las cantidades de capital y trabajo que renta el hogar representativo $\left(L_{F}=L_{H}=L, K_{F}=K_{H}=K\right)$. 
La introducción de $r_{K}$ no debe parecer extraña; Barro \& Sala-i-Martin (2009) hacen hincapié en este término al denominarlo en forma correcta como una tasa de rendimiento. Es importante no confundir los conceptos de precio de renta y de tasa de rendimiento, ya que ambos representan dos observables de distinta naturaleza. Los precios sólo convierten stocks en stocks, mientras que las tasas transforman stocks en flujos.

Asimismo, para conservar la simetría dimensional en las relaciones Eq. (7) y Eq. (8), el factor trabajo $L$ puede ser reformulado en términos de la variación de una nueva variable, $P$, de tipo stock $^{17}$ :

$$
L \equiv \frac{d P(t)}{d t}=\dot{P}(t)=n P(t)
$$

La cual se asume que crece de forma exponencial y por tanto a una tasa de crecimiento constante $(\dot{P}(t) / P(t))=n^{18}$.

La variable $P$ se denominará stock de población y se debe interpretar como el número de habitantes o agentes económicos dentro del hogar representativo. Por ende, la dimensión asociada a dicha variable es cantidad de población $[P]=Q_{P}$. Esta reformulación del factor trabajo es congruente desde el punto de vista dimensional. La introducción del operador derivada no contraviene las dimensiones originales de $L$ :

$$
[L]=\frac{Q_{P}}{T}=\left[\frac{d P(t)}{d t}\right]
$$

17 Esta reformulación está sustentada en la hipótesis de que, en una situación de equilibrio del mercado, todo el trabajo es empleado. Es decir, cada habitante otorga exactamente una unidad de trabajo, independientemente del precio asociado a dicho factor, i.e. $Q_{P}=Q_{L}$.

18 En ocasiones, para simplificar la notación no se hará explícita la dependencia temporal de las variables. Cuando se realicen operaciones de derivación respecto del tiempo se utilizará la notación de punto de Newton. Cuando la derivación sea respecto de las variables en el argumento se utilizará la notación de comas. 
Asimismo, la introducción de la variable $P$ permite transformar correctamente las variables originales en variables per cápita.

$$
k(t) \equiv \frac{K(t)}{P(t)}, \quad n \equiv \frac{L(t)}{P(t)}, \quad f(k ; n) \equiv \frac{F(K ; L)}{P(t)}, \quad y(t) \equiv \frac{Y(t)}{P(t)}
$$

Adviértase que al introducir la Eq. (9) dentro de la función de producción, se obtiene que la función no sólo depende del capital físico per cápita, sino que también dependerá de la tasa de crecimiento de $P(\mathrm{t})$. Este hecho proviene simplemente de la propiedad de homogeneidad de la función de producción ${ }^{19}$.

Para determinar exactamente el efecto de las modificaciones realizadas en las ecuaciones Eq. (7), Eq. (8), y la introducción de $P(t)$ en la función de producción, se procede a calcular de forma explícita la función $f(k ; n)$. Partiendo de la Eq. (7) se reescribe la ecuación en variables per cápita y se deriva respecto del tiempo:

$$
\dot{y}(t)=\dot{r}(t) k(t)+r(t) \dot{k}(t)+\dot{w}(t) n
$$

Desarrollando la expresión

$$
\begin{gathered}
\frac{\dot{y}(t)}{y(t)}=\frac{r(t) k(t)}{y(t)}\left(\frac{\dot{r}(t)}{r(t)}\right)+\frac{r(t) k(t)}{y(t)}\left(\frac{\dot{k}(t)}{k(t)}\right)+\frac{w(t) n}{y(t)}\left(\frac{\dot{w}(t)}{w(t)}\right) \\
\frac{\dot{y}}{y}=\beta\left(\frac{\dot{r}}{r}\right)+\beta\left(\frac{\dot{k}}{k}\right)+(1-\beta)\left(\frac{\dot{w}}{w}\right)
\end{gathered}
$$

Donde se ha definido $\beta(t) \equiv \frac{r(t) k(t)}{y(t)}$ como la participación del capital en la producción, $(1-\beta(t)) \equiv \frac{w(t) n}{y(t)}$ como la participación del trabajo.

19 Un requisito que debe cumplir cualquier función de producción es que presente rendimientos constantes a escala. Dicho de otra forma, la función debe ser una función homogénea de grado uno en cada uno de sus argumentos. Ver Acemoglu (2009:29). 
Si se considera la hipótesis de que las participaciones del capital y el trabajo permanecen constantes, i.e. $\beta(t)=\alpha$, y el rendimiento del capital y el precio del trabajo permanecen invariables, i.e. $\dot{r}=\dot{w}=0$, entonces la Eq. (11) puede integrarse directamente. El resultado final es la función de Cobb-Douglas:

$$
y(t)=f(k ; n)=a_{0} k^{\alpha}, \quad Y(t)=F(K ; L)=a_{0} K^{\alpha}\left(\frac{L}{n}\right)^{1-\alpha}
$$

Con $a_{0}$ una constante de integración. Con este cálculo se validan las modificaciones realizadas en la Eq. (7) y en la Eq. (8), ya que se ha obtenido de forma explícita la función de producción de Cobb-Douglas. Asimismo, queda justificada la nueva representación del trabajo, Eq. (9), como la variación del stock de población y su incorporación dentro de la función de producción.

\section{Análisis dimensional del modelo Neoclásico}

En esta sección se analiza la consistencia dimensional del modelo Neoclásico y se incorporan las correcciones que se proponen en la segunda sección.

La versión básica del modelo Neoclásico se construye en el contexto de una Economía cerrada, en la cual existe un solo mercado donde se produce y consume un solo bien; es decir, este bien puede transformarse ya sea en un insumo de producción (por ejemplo, capital físico) o en un bien de consumo. Igualmente, se asume que el principio de agregación es válido (existencia de un agente representativo) y por lo tanto el problema general de optimización de todos los agentes que participan en el mercado puede reducirse a resolver un solo problema de optimización ${ }^{20}$.

La principal diferencia del modelo Neoclásico respecto a los primeros modelos de crecimiento, por ejemplo el modelo de Solow-Swan, es que el modelo Neoclásico considera de forma explícita el consumo del

20 Para una explicación más detallada sobre el modelo Neoclásico, se recomienda revisar: Cass (1965), Samuelson (1970) y Acemoglu (2009). 
hogar representativo y de esta forma se endogeneiza el ahorro, el cual se consideraba una variable exógena en los modelos anteriores.

En el modelo Solow-Swan se asume que la inversión (en el contexto de una Economía cerrada y de un mercado de competencia perfecta) es igual a la función de ahorro, y que la función de ahorro es igual a su vez a una proporción fija (exógena) del ingreso (Solow, 1956). En el modelo Neoclásico esta suposición no se realiza más. El ahorro del hogar ahora está descrito explícitamente en términos del ingreso $Y(t)$ y el consumo $C(t)$ :

$$
I(t)=S(t)=Y(t)-C(t)=r_{K}(t) K(t)+w_{L}(t) L(t)-C(t)
$$

Así, la ecuación que describe la acumulación de capital del hogar representativo está dada por:

$$
\frac{d K(t)}{d t}=I(t)=r_{K}(t) K(t)+w_{L}(t) L(t)-C(t)
$$

Para reducir el número de variables involucradas en la Eq. (14) y obtener una ecuación diferencial en una variable, se introducen variables per cápita. Así ${ }^{21}$ :

$$
\dot{k}(t)=r_{K}(t) k(t)+n w_{L}(t)-c(t)-n k(t)
$$

Por otra parte, la condición de maximización del beneficio de la empresa implica que, en el equilibrio de mercado, la función de producción deba satisfacer las siguientes condiciones (Acemoglu, 2009:33):

$$
\frac{\partial F}{\partial K}=r_{K}(t)+\delta, \quad \frac{\partial F}{\partial L}=w_{L}(t)
$$

Dichas condiciones pueden ser reescritas en términos de variables per cápita:

$$
f^{\prime}(k ; n)=r_{K}(t)+\delta, \quad f(k ; n)-k f^{\prime}(k ; n)=n w_{L}(t)
$$

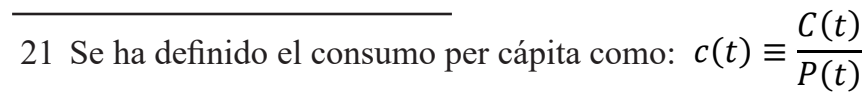


Introduciendo estas condiciones en la Eq. (15), se obtiene la ecuación de Solow-Swan modificada:

$$
\dot{k}(t)=f(k ; n)+(n+\delta) k(t)-c(t)
$$

La ecuación Eq. (16) es similar a la ecuación original que se muestra en la literatura (Ver Acemoglu (2009), Barro y Sala-i-Martin (2009) o Solow (1956), excepto por la diferencia que presenta la función de producción per cápita, ya que ahora la función depende explícitamente de la tasa de crecimiento $n$.

El objetivo de la Eq. (16) es describir la acumulación del capital físico per cápita en función de dos factores: la función de producción $f(k$; $n)$ y el consumo per cápita $c(t)$. Para resolver esta ecuación y estudiar la dinámica del capital físico es necesario definir la forma explícita de la función de producción y proponer una dinámica para el consumo. Es en este contexto que el modelo se interpreta como un problema de control óptimo ${ }^{22}$.

En el caso del modelo Neoclásico, lo que se busca optimizar (maximizar) es el bienestar de la sociedad en un intervalo de tiempo (también llamado horizonte de planeación). La hipótesis fundamental del modelo es que el bienestar de la sociedad en cada instante del horizonte de planeación puede modelarse a través de una función de utilidad, la cual depende en primera instancia del nivel de consumo solamente. De esta manera, el modelo considera admisible suponer la existencia de un solo agente (hogar representativo) que muestre todas las decisiones económicas que se realizan.

22 En general, los problemas de control óptimo buscan optimizar un funcional de costo $J$, el cual depende de las variables de estado $x_{i}(t)$, mismas que describen la dinámica del sistema, y de las variables de control $u_{i}(t)$. Estas variables pueden controlarse o elegirse de tal forma que optimicen el funcional de costo. 
Este agente busca optimizar su bienestar, que es representado por una función de utilidad $U(t)$ que depende exclusivamente del consumo del agente ${ }^{23}$ :

$$
u(t)=u(c(t))
$$

Sin embargo, la optimización se realiza en forma intertemporal considerando el flujo futuro de utilidad. Por lo tanto, el agente optimiza el valor presente de su utilidad en un horizonte de tiempo infinito $[0, \infty]$; es decir, maximiza la siguiente expresión:

$$
u_{P}=\int_{0}^{\infty} u(c(t)) e^{-\rho t} d t
$$

El funcional de costo, Eq. (18), depende únicamente de dos variables, la variable de estado $k(t)$ y la variable de control $c(t)$. La presencia del multiplicador $e^{-\rho t}$ se debe al descuento del valor futuro de la utilidad, el cual se realiza con una tasa de descuento subjetiva $\rho$ estrictamente mayor a cero $(\rho>0)$; dicha condición garantiza que el funcional de costo, Eq. (18), sea convergente y, asimismo, garantiza la existencia de las soluciones del problema de optimización (Acemoglu, 2009:252).

Por otra parte, el problema de optimización está sujeto a satisfacer la evolución de la variable de estado. En este caso, como la variable de estado es el capital físico per cápita $k$, la optimización estará sujeta a satisfacer la ecuación dinámica del capital, i.e. la ecuación de Solow-Swan modificada Eq. (16) (Cass, 1965).

En síntesis, el modelo Neoclásico considera que el problema de crecimiento económico puede tratarse como un problema de control óptimo al asumir que el agente representativo busca maximizar su utilidad presente:

$$
\max u_{P}=\max \left[\int_{0}^{\infty} u(c(t)) e^{-\rho t} d t\right]
$$

23 Se ha definido la función de utilidad en términos de variables per cápita: $u(c(t)) \equiv \frac{U(C(t))}{P(t)}$ 
Sujeto a la restricción de la acumulación de capital, Eq. (16):

$$
\dot{k}(t)=f(k ; n)-(n+\delta) k(t)-c(t)
$$

Una vez especificadas las bases del modelo Neoclásico, se realiza su análisis dimensional. Calculando primero las dimensiones de cada término de la ecuación Eq. (19):

$$
\begin{gathered}
{\left[u_{P}\right]=(U)\left(\frac{1}{Q_{P}}\right)=\frac{U}{Q_{P}}} \\
{\left[u(c(t)) e^{-\rho t} d t\right]=[u(c(t))]\left[e^{-\rho t}\right][d t]=\left(\frac{U}{Q_{P} T}\right)\left(1_{D}\right)(T)=\left(\frac{U}{Q_{P}}\right)}
\end{gathered}
$$

De esta manera:

$$
\left[u_{P}\right]=\frac{U}{Q_{P}}=\left[u(c(t)) e^{-\rho t} d t\right]
$$

Por consiguiente, el funcional de costo no presenta ninguna inconsistencia dimensional, ya que al integrar el flujo de utilidad $u(c(t))$ en el tiempo se transforma dicho término en un stock de utilidad. De igual forma, el término de descuento no introduce ningún problema, debido a que las dimensiones del argumento de la exponencial se anulan y el término en su conjunto es adimensional.

Se prosigue con el cálculo de las dimensiones de cada término de la ecuación de restricción Eq. (20):

$$
\begin{gathered}
{[\dot{k}]=\left(\frac{Q_{K}}{Q_{P}}\right)\left(\frac{1}{T}\right)=\frac{Q_{K}}{Q_{P} T}, \quad[f(k ; n)]=\left(\frac{1}{Q_{P}}\right)\left(\frac{Q_{K}}{T}\right)=\frac{Q_{K}}{Q_{P} T}} \\
{[(n+\delta) k]=\left(\frac{1}{T}\right)\left(\frac{Q_{K}}{Q_{P}}\right)=\frac{Q_{K}}{Q_{P} T}, \quad[c]=\left(\frac{1}{Q_{P}}\right)\left(\frac{Q_{K}}{T}\right)=\frac{Q_{K}}{Q_{P} T}} \\
{[\dot{k}(t)]=\left(\frac{Q_{K}}{Q_{P} T}\right)=[f(k ; n)-(n+\delta) k(t)-c(t)]=\left(\frac{Q_{K}}{Q_{P} T}\right)-\left(\frac{Q_{K}}{Q_{P} T}\right)-\left(\frac{Q_{K}}{Q_{P} T}\right)} \\
{[\dot{k}(t)]=[f(k ; n)-(n+\delta) k(t)-c(t)]}
\end{gathered}
$$

El cálculo de las dimensiones de la Eq. (20) muestra que ésta es dimensionalmente congruente. Es importante remarcar que la consistencia 
dimensional de las ecuaciones Eq. (19) y Eq. (20) queda garantizada cuando definimos las variables per cápita respecto al stock de población.

¿Qué efecto tiene en el modelo las modificaciones realizadas? Para identificar los efectos se debe proceder a resolver el modelo. La solución del problema de control óptimo se realiza mediante la metodología del Principio Máximo de Pontryagin ${ }^{24}$. Dicho principio establece un conjunto de condiciones necesarias para que las trayectorias solución de las variables de estado y de control puedan considerarse óptimas. Estas condiciones se expresan en términos de una función, que se conoce como el Hamiltoniano de Pontryagin.

En el caso del modelo Neoclásico, se cuenta con sólo una variable de estado, que es el stock de capital físico per cápita $k(t)$, una variable de control, que es el consumo per cápita $\mathrm{c}(t)$ y una sola función restricción, que es la ecuación de acumulación del capital, Eq. (20). Así, el Hamiltoniano asociado al modelo es el siguiente:

$$
H(k, c, p, t)=u(c) e^{-\rho t}+p(t)(f(k ; n)-(n+\delta) k(t)-c(t))
$$

Donde $p(t)$ denota la variable de coestado ${ }^{25}$. Dado que la función objetivo del modelo depende explícitamente del tiempo, debido al factor de descuento $e^{-p \mathrm{t}}$, el modelo debe ser transformado a un problema de optimización autónomo mediante la definición de un Hamiltoniano autónomo (también llamado de tiempo corriente):

$$
\widehat{H}=H e^{\rho t}
$$

De igual forma, se define una variable de coestado en tiempo corriente:

$$
\mu(t)=p(t) e^{\rho t}
$$

24 Para ver con mayor profundidad dicha metodología, se recomienda revisar Acemoglu (2009:227-275) y Pedregal (2004:195-224).

25 La variable de coestado se interpreta como el precio sombra del stock de capital. Es decir, es el valor de una unidad de capital en términos de la utilidad. Por ende, las dimensiones de dicha variable son: $[p]=U / Q_{K}$. 
Por consiguiente, reescribiendo Eq. (22) se obtiene:

$$
\widehat{H}(k, c, p, t)=u(c)+\mu(t)(f(k ; n)-(n+\delta) k(t)-c(t))
$$

Para que las trayectorias de las variables de estado $k(t)$ y control $c(t)$ sean óptimas y satisfagan la ecuación de restricción, el Hamiltoniano en tiempo corriente debe satisfacer las condiciones (Ver Acemoglu, 2009:235):

$$
\begin{gathered}
\frac{\partial \widehat{H}}{\partial c}=0=u^{\prime}(c)-\mu(t) \\
\dot{k}(t)=\frac{\partial \widehat{H}}{\partial \mu}=f(k ; n)-(n+\delta) k(t)-c(t) \\
\dot{\mu}(t)=-\frac{\partial \widehat{H}}{\partial k}+\rho \mu(t)=-\mu(t)\left(f^{\prime}(k ; n)-(n+\delta)\right)+\rho \mu(t)
\end{gathered}
$$

Las ecuaciones (25-27) forman el sistema de ecuaciones diferenciales cuya solución determinará las soluciones óptimas de consumo y de capital físico per cápita.

Antes de continuar con la solución del modelo, se analizan las dimensiones de las ecuaciones anteriores:

$$
\begin{gathered}
{[\widehat{H}]=\frac{U}{Q_{P} T}=[u(c)]=[\mu(t)][f(k ; n)]=[\mu(t)][(n+\delta) k(t)]=[\mu(t)][c(t)]} \\
{\left[\frac{\partial \widehat{H}}{\partial c}\right]=\left(\frac{U}{Q_{K}}\right)=\left[u^{\prime}(c)\right]=[\mu(t)]} \\
{[\dot{k}(t)]=\left(\frac{Q_{K}}{Q_{P} T}\right)=\left[\frac{\partial \widehat{H}}{\partial \mu}\right]} \\
{[\dot{\mu}(t)]=\left(\frac{U}{Q_{K} T}\right)=\left[\frac{\partial \widehat{H}}{\partial k}\right]=[\rho \mu(t)]}
\end{gathered}
$$

Los resultados del análisis dimensional del Hamiltoniano y de las relaciones (25-27) permiten señalar que dichas ecuaciones son dimensio- 
nalmente consistentes. Resolviendo el sistema de ecuaciones (25-27), se reescriben las ecuaciones de la siguiente manera:

$$
\begin{gathered}
u^{\prime}(c)=\mu(t) \\
\dot{\mu}(t)=\mu(t)\left(\rho+n+\delta-f^{\prime}(k ; n)\right) \\
\dot{k}(t)=f(k ; n)-(n+\delta) k(t)-c(t)
\end{gathered}
$$

Luego, con el propósito de obtener un sistema de sólo dos ecuaciones diferenciales, se elimina la relación Eq. (28) al introducirla en las otras dos relaciones (29-30). Por consiguiente, se obtiene la ecuación dinámica para el consumo per cápita:

$$
\dot{c}(t)=\frac{c(t)}{\varepsilon_{u}(c)}\left(f^{\prime}(k ; n)-(\rho+n+\delta)\right)
$$

Donde el término $\varepsilon_{u}$ representa la elasticidad marginal de la función de utilidad ${ }^{26}$. Asimismo, se introduce explícitamente la función de Cobb-Douglas (ver la ecuación Eq. (12)) en Eq. (30) para expresar en forma completa la ecuación dinámica del capital:

$$
\dot{k}(t)=a_{0} k^{\alpha}-(n+\delta) k(t)-c(t)
$$

Las ecuaciones (31) y (32) determinan las trayectorias óptimas de consumo y capital físico per cápita del problema de control óptimo del modelo Neoclásico modificado. La equidimensionalidad del modelo

26 Hay que considerar que los coeficientes de elasticidad son números adimensionales, y en este caso la adimensionalidad de la elasticidad marginal de la utilidad se verifica rápidamente con un poco de álgebra:

$$
\left[\varepsilon_{u}(c)\right]=\left(\frac{U T^{2} Q_{P}^{2}}{Q_{K}^{2}}\right)\left(\frac{Q_{K}}{Q_{P} T}\right)\left(\frac{U T Q_{P}}{Q_{K}}\right)^{-1}=1
$$


modificado se corrobora con el análisis de las dimensiones de Eq. (31) y Eq. (32):

$$
\begin{gathered}
{[\dot{k}(t)]=\left(\frac{Q_{K}}{Q_{P} T}\right)=\left[a_{0} k^{\alpha}\right]=[(n+\delta) k(t)]=[c(t)]} \\
{[\dot{c}(t)]=\left(\frac{Q_{K}}{Q_{P} T^{2}}\right)=\left[\frac{c(t)}{\varepsilon_{u}(c)} f^{\prime}(k ; n)\right]=\left[\frac{c(t)}{\varepsilon_{u}(c)}(\rho+n+\delta)\right]}
\end{gathered}
$$

Un análisis exhaustivo de las propiedades dinámicas del modelo con la corrección propuesta queda fuera de las posibilidades de este artículo. No obstante, se puede mencionar que la forma más adecuada de analizar las trayectorias óptimas, Eq. (31) y Eq. (32), es a través del análisis del espacio fase, ya que dicho análisis permite visualizar en forma global el comportamiento dinámico de las trayectorias, así como el análisis de estabilidad de los puntos de equilibrio del sistema, que proporciona las características fundamentales de la dinámica ${ }^{27}$.

El análisis desarrollado a lo largo de esta última sección muestra que el modelo Neoclásico cumple con el principio de homogeneidad dimensional, siempre y cuando se acepten las modificaciones realizadas a las ecuaciones de ingreso, Eq. (7), y beneficio, Eq. (8). Asimismo, la introducción de las variables per cápita en términos del stock de población es un requisito necesario, ya que de otra forma el modelo fallaría en cumplir el principio de homogeneidad al no relacionar de forma adecuada la tasa de cambio del capital per cápita con la función de producción.

Las implicaciones teóricas del modelo Neoclásico corregido (equidimensional) aún no han sido advertidas completamente, ni se han comparado con los resultados del modelo original. Sin embargo, es probable que las implicaciones del modelo equidimensional sean más relevantes para entender la dinámica del crecimiento económico, ya que estarán libres de la inconsistencia dimensional del modelo original.

27 Para ver con mayor profundidad dichos métodos, se recomienda revisar Barro \& Sala-i-Martin (2009) o Shone (2002). 


\section{Conclusiones}

La teoría económica se ha esforzado por tener el mismo nivel de éxito en la comprensión y predicción de los fenómenos económicos que el éxito alcanzado por la ciencia en la comprensión y predicción de los fenómenos naturales. Por esa razón, la teoría económica ha emulado los métodos analíticos, estadísticos y matemáticos de las ciencias naturales, acotándose a sí misma, en alto grado, a la formulación de modelos matemáticos para explicar la realidad económica. Es decir, esta teoría tiende a convertirse en un ejercicio de modelación matemática más que en una verdadera disciplina científica de observación. De esta manera, aunque los economistas afirman que la Economía es una ciencia objetiva y sólida al igual que las ciencias naturales. Actualmente la disciplina está construida sobre teorías, supuestos y modelos matemáticos que poseen puntos endebles y cuestionables desde un punto de vista analítico y teórico, tal y como lo ha intentado demostrar la presente investigación.

Al hacer hincapié en la idealización de la técnica y la elegancia formal de la matemática, la teoría económica se ha convertido en una suerte de matemáticas sociales que emplea términos económicos. Esta tendencia de la Economía hacia el formalismo ha tenido consecuencias; se ha sobrevalorado la formalidad y la elegancia matemática en detrimento del contenido, la consistencia y el argumento. Se atiende más a la forma en que una teoría económica o hipótesis es presentada y se descuida su contenido (Blaug, 1998). Además, los resultados de dicho programa de formalización matemática han sido en el mejor de los casos insuficientes o inadecuados.

Comúnmente se cree que cuando se diseña o utiliza un modelo económico-matemático se hace Economía, pero se olvida que la mayoría de las relaciones propuestas en un modelo son relaciones de naturaleza matemática, aunque con un contenido o significado económico. Todas las inferencias son obtenidas matemáticamente y poca atención se dá a si esas variables, conceptos y relaciones funcionales tienen alguna semejanza o correlación con alguna observación del mundo económico. Al no atender este hecho fundamental, los economistas han fallado en emu- 
lar los métodos de las ciencias naturales en un aspecto crucial, el cual es considerar el correcto uso de las dimensiones. Esto lleva a una conclusión definitiva que es necesario señalar para juzgar apropiadamente el actual papel de la modelación económica: ciertas partes de la teoría económica pueden contener fallas analíticas significativas, y dichas fallas son transmitidas a los modelos que se sustentan en dicha teoría.

En este trabajo se ha demostrado cómo el Análisis Dimensional puede ser aplicado a los modelos de crecimiento económico; mediante el Análisis Dimensional del modelo Neoclásico, se obtuvo un nuevo sistema de ecuaciones diferenciales a partir del modelo corregido -las ecuaciones (31-32) - el cual carece de la inconsistencia dimensional del modelo original. No obstante, se ha pagado un precio en cuestión de simplicidad, ya que ahora las ecuaciones que describen la dinámica de las trayectorias óptimas de consumo y acumulación de capital se han vuelto más complejas, dada la incorporación de la tasa de crecimiento de la población $\mathrm{n}$ dentro de las funciones de producción y de utilidad.

La incorporación de la tasa de crecimiento de la población no impide analizar de forma inmediata la dinámica de equilibrio del modelo; sin embargo, si esta tasa de crecimiento dejara de ser constante y dependiera de otros factores, la complejidad analítica del modelo se incrementaría significativamente e impediría aplicar los métodos clásicos de análisis de estabilidad ${ }^{28}$.

Empero estas implicaciones, la dinámica que presenta el nuevo sistema de ecuaciones equidimensionales del modelo pudiera ser más rica y compleja en relación al comportamiento de la acumulación del capital físico per cápita, ya que ahora esta acumulación dependerá directamente de la dinámica de la tasa de crecimiento de la población $\mathrm{n}$, lo cual introduciría una fuerte no linealidad en la dinámica del modelo. Como se hizo mención previamente, un análisis exhaustivo de las propiedades

28 A diferencia del modelo Neoclásico original, en el cual se puede realizar con relativa sencillez un análisis de estabilidad local o global. Ver Acemoglu (2009) o Barro \& Sala-i-Martin (2009). 
dinámicas del modelo corregido que se propone queda fuera de las posibilidades de este artículo y será materia de una investigación futura.

Por último, se indican las posibles líneas de investigación que se pueden desarrollar en torno a los temas tratados en este trabajo. Se considera necesario analizar a profundidad los fundamentos microeconómicos y macroeconómicos de la teoría económica convencional, con el objetivo de identificar si la omisión del Análisis Dimensional es un suceso aislado u ocurre de forma sistemática en la teoría. Asimismo, se debe estudiar con mayor profundidad los principios del Análisis Dimensional con el propósito de enfocarlos al campo de la Economía, ya que sobre este punto aún quedan varias cuestiones teóricas por responder.

\section{Bibliografía}

Acemoglu, D. Introduction to Modern Economic Growth. Princeton University Press, New York, 2009, pp. 990.

Baiocchi, G. "On dimensions of ecological economics", Ecological Economics, Volumen 74, 2012, pp. 1-9.

Balaguer, P. Application of Dimensional Analysis in Systems Modelling and Control Design, The Institution of Engineering and Technology, London, 2013, pp. 142.

Barnet II, W. "Dimensions and Economics: Some Problems", The Quarterly Journal of Austrian Economics, Volumen 7, 2004, pp. 95-104.

Barro, R. J. \& Sala-i-Martin, X. Crecimiento Económico, Editorial Reverte, México D.F, 2009, pp. 654.

Blaug, M. "The problems with formalism, interview with Mark Blaug", Challange, 41(3), 1998, pp. 35-45.

Bunge, M. "A Mathematical Theory of Dimensions and Units of Physical Quantities". En: M. Bunge \& et al, edits. Problems in the Foundations of Physics, Springer-Verlag, Montreal, 1971, pp. 162.

Carlson, D. D. "A Mathematical Theory of Physical Units, Dimensions and Measures", Rational Mechanics and Analysis, Volumen 70, 1979, pp. 289-304. 
Cass, D. "Optimum Growth in an Aggregative Model of Capital Accumulation”, The Review of Economic Studies, 32(3), 1965, pp. 233-240.

De Jong, F. \& Kumar, K. "Some Considerations on a Class of Macro-Economic Production Functions", The Economist, Volumen 120, 1972, pp. 134-152.

Einstein, A. Essays in Science, Philosophical Library, New York, 1933, pp. 112.

Grudzewski, W. M. \& Roslanowska, K. Application of Dimensional Analysis in Economics, IOS Press, Amsterdam, 2013, pp. 220.

Macagno, E. O. "Historico-critical review of Dimensional Analysis", Journal of The Franklin Institute, Volumen 292, 1971, pp. 391-402.

Okishio, N. "Dimensional Analysis in Economics", Kobe University economic review, Volumen 28, 1982, pp. 31-44.

Pedregal, P. Introduction to Optimization, Springer-Verlag, New York, 2004, pp. 245.

Pindyck, R. S. \& Rubinfeld, D. L. Microeconomía, 7th ed, Pearson Prentice Hall, Madrid, 2009, pp. 850.

Ramsey, F. P. “A Mathematical Theory of Saving”, The Economic Journal, 38(152), 1928, pp. 543-559.

Samuelson, P. A. Maximum Principles in Analytical Economics, Nobel Memorial Lectures, New York, 1970, pp. 1-15.

Shone, R. Economic Dynamics, Cambridge University Press, Boston, 2002, pp. 708.

Solow, R. M. "A Contribution to the Theory of Economic Growth", Quarterly Journal of Economics, 70(1), 1956, pp. 65-94.

Sonin, A. A. The Physical Basis of Dimensional Analysis, MIT Press, Boston, 2001, pp.57.

White, F. M. Fluid Mechanics 7th ed, McGraw Hill, New York, 2011, pp. 862. 